\title{
Antimicrobial susceptibility of Actinobacillus pleuropneumoniae strains isolated from pig's clinical samples in Serbia
}

\author{
JADRANKA ŽUTIĆ, LJILJANA PAVLOVIĆ*, OLIVER RADANOVIĆ, \\ VLADIMIR RADOSAVLJEVIĆ, BRANISLAV KURELJUŠIĆ, DRAGICA VOJINOVIĆ
}

\author{
Scientific Institute of Veterinary Medicine of Serbia, ul. Vojvode Toze 14, 11000 Belgrade, Serbia \\ *Institute of Public Health of Serbia, Center for Microbiology, ul. Dr Subotica 5, 11000 Belgrade, Serbia
}

\section{Žutić J., Pavlović L., Radanović O., Radosavljević V., Kureljušić B., Vojinović D. Antimicrobial susceptibility of Actinobacillus pleuropneumoniae strains isolated from pig's clinical samples in Serbia}

\section{Summary}

Porcine pleuropneumonia, caused by Actinobacillus pleuropneumoniae is one of the most important bacterial diseases of the pig respiratory tract. One hundred and forty eight Actinobacillus pleuropneumoniae strains isolated from pig's clinical samples were tested for their susceptibility to 11 antimicrobials by disk diffusion method. Isolates were obtained from the pathoanatomically altered pig's lung that died with signs of respiratory disease. Antimicrobial discs with following concentration were used: ampicillin $10 \mu \mathrm{g}$, amoxicillin $10 \mu \mathrm{g}$, amoxicillin/clavulanic acid 20/10 $\mu \mathrm{g}$, cefotaxime $30 \mu \mathrm{g}$, gentamicin $10 \mu \mathrm{g}$, penicillin $10 \mathrm{IU}$, streptomycin $10 \mu \mathrm{g}$, tetracycline $30 \mu \mathrm{g}$, enrofloxacin $5 \mu \mathrm{g}$, lincospectin $15 / 200 \mu \mathrm{g}$, florfenicol $30 \mu \mathrm{g}$ and trimethoprim/sulfamethoxazole $\mathbf{1 . 2 5} / \mathbf{2 3 . 7 5} \mu \mathrm{g}$. Isolated strains showed various susceptibility to tested antimicrobials. Seventy-five percent of isolates were resistant to one or more drugs frequently used in the treatment of pig's diseases at farms in Serbia. All 148 isolates were susceptible to cefotaxime, enrofloxacin and florfenicol, while $96 \%$ of them were susceptible to amoxicillin/clavulanic acid and lincospectin. Susceptibility of isolates to amoxicillin, gentamicin and trimethoprim/sulfamethoxazole were $91 \%, 77 \%$ and $64 \%$, respectively. The highest resistance rates were determined for tetracycline and streptomycin, $34 \%$ and $31 \%$, respectively. When compared to our previous results, we noticed a decrease in resistance to tetracyclines and trimethoprim/sulfamethoxazole. Our results indicate that A. pleuropneumoniae strains isolated from pig's clinical samples in Serbia show high susceptibility to antimicrobial agents which are most often used in the pig industry.

Keywords: Actinobacillus pleuropneumoniae, antimicrobial susceptibility, pig

Porcine pleuropneumonia, caused by Actinobacillus pleuropneumoniae (A. pleuropneumoniae), is one of the most important bacterial diseases of the pig respiratory tract. This highly contagious respiratory disease is widely spread among pig-keeping countries causing major economic losses in the swine industry $(21,30)$. The most important $A$. pleuropneumoniae virulence factors include surface polysaccharides (18), iron uptake systems (4), Apx toxins (11, 16, 28), components of anaerobic metabolism (17), and outer membrane proteins $(8,12)$. On the basis of the $\beta$-nicotinamide adenine dinucleotide $(\beta$-NAD) requirement for growth $A$. pleuropneumoniae has been classified into biovar 1 ( $\beta$-NAD dependent) and biovar 2 ( $\beta$-NAD independent). Biovar 2 can synthesize NAD in the presence of specific pyridine nucleotides or their precursors $(6,21)$. Each biovar is further divided into serovars. Fifteen serovars of both biovars $A$. pleuropneumoniae based on capsular and lipopolysaccharide antigens have been described, with a variation in virulence and regional distributions. There are 13 serotypes of biotype 1 , and 2 serotypes of biotype 2 . Certain strains of biovar 2 have the same antigenic determinants as serovars 2, 4, 7 and 9 of biovar 1 (5, 21). The four major RTX toxins ApxI, ApxII, ApxIII and ApxIV are cytolytic and/or hemolytic, and secreted by the different serotypes in various combinations (11). Compared to ApxI, ApxII and ApxIII, ApxIV is produced by all serotypes of $A$. pleuropneumoniae, highly specific to $A$. pleuropneumoniae and expressed only in vivo but not in vitro conditions (28). Most of the pathological consequences of porcine pleuropneu- 
monia can be attributed to these Apx toxins (16). The role of biofilms in the pathogenicity of A. pleuropneumoniae is also very important (29). Cells of A. pleuropneumoniae in biofilm were 100 to 30,000 times more resistant to antimicrobials than their planktonic counterparts (3). The disease is transmitted by aerosol within short distances or by direct contact with infected pigs. Clinical signs are fever, severe respiratory distress with cyanosis, coughing and epistaxis. Characteristic pathoanatomic alterations are fibrinohemorhagic necrotizing broncho-pneumonia and fibrinous pleuritis (13). Growing pigs are most likely to be affected when they are 12-16 weeks old, but the disease can occur in all ages of swine. Efficient antimicrobial therapy is an important protective measure for the control and prevention of porcine pleuropneumonia $(3,20)$. A broad range of antimicrobial agents used for the treatment of disease including $\beta$-lactams (penicillin, amoxicillin, ampicillin, and ceftiofur), fluoroquinolones, gentamicin, trimethoprim/sulfamethoxazole, tiamulin, lincomycin/spectinomycin, and tetracyclines $(25,30)$. Isolates of $A$. pleuropneumoniae are generally reported to be susceptible toward fluoroquinolones, ceftiofur and florfenicol, but various antimicrobial resistance patterns have been observed to others antimicrobials in different countries $(10,32)$.

The last research of the antimicrobial susceptibility of A. pleuropneumoniae in Serbia was published seven years ago (34), but the current situation is unknown. Therefore, the aim of our study was to investigate the antimicrobial susceptibility of $A$. pleuropneumoniae strains isolated from clinical samples originating from pig breeding farms in Serbia in order to provide adequate treatment of infected animals and thus prevent the spread of disease in swine population.

\section{Material and methods}

Bacterial strains. A. pleuropneumoniae isolates were obtained from the pathoanatomically altered pig's lung that died with signs of respiratory disease. A total of 148 clinical isolates were tested during the period from 2014-2015 $(2014-89,2015-59)$ from 9 large pig breeding farms located in northwest Serbia. Samples of pig lungs were inoculated on $5 \%$ sheep blood agar with Staphylococcus aureus as a source of $\mathrm{NAD}$, and incubated in a jar with reduced oxygen and enhanced carbon dioxide content. Suspicious colonies were subcultivated on chocolate agar with PolyVitex (bioMerieux, France) and sheep blood agar, as before. A. pleuropneumoniae were identified on the basis of the Gram-stains, haemolysis on 5\% sheep blood agar, positive CAMP test, requirement for NAD and urease production.
Antimicrobial susceptibility testing. Susceptibility to antimicrobials of $A$. pleuropneumoniae isolates was determined by the disk diffusion method using chocolate Mueller-Hinton agar (Mueller Hinton II agar, HiMedia, India) according to CLSI recommendation (8). The diameter of the inhibition zone of the individual drug was determined after $20-22$ hours of incubation at $37^{\circ} \mathrm{C}$. Isolates were classified as susceptible, intermediate or resistant according to the CLSI and manufacturer's instructions. A. pleuropneumoniae ATCC 27090 was used as a quality control strain. Antimicrobial discs with following concentration were used: ampicillin $10 \mu \mathrm{g}$, amoxicillin $10 \mu \mathrm{g}$, amoxicillin/clavulanic acid $20 / 10 \mu \mathrm{g}$, cefotaxime $30 \mu \mathrm{g}$, gentamicin $10 \mu \mathrm{g}$, penicillin $10 \mathrm{IU}$, streptomycin $10 \mu \mathrm{g}$, tetracycline $30 \mu \mathrm{g}$, enrofloxacin $5 \mu \mathrm{g}$, lincospectin $15 / 200 \mu \mathrm{g}$, florfenicol $30 \mu \mathrm{g}$ and trimethoprim/sulfamethoxazole 1.25/23.75 $\mu \mathrm{g}$ (Rosco Diagnostica, Denmark; Bioanalyse, Turkey).

\section{Results and discussion}

A. pleuropneumoniae clinical strains isolated in our study showed various susceptibility to the tested antimicrobials. The results are shown in Tab. 1.

Seventy-five percent of isolates were resistant to one or more of the antimicrobials frequently used in the treatment of pig's diseases at farms in Serbia. All 148 isolates were susceptible to cefotaxime, enrofloxacin and florfenicol, while $96 \%$ of them were susceptible to amoxicillin/clavulanic acid and lincospectin. Similar results were obtained in other studies for florfenicol $(14,32)$, ceftiofur/cefotaxime (19) and enrofloxacin (24). A high susceptibility to lincospectin correlates with the observed susceptibility of $95 \%$ in neighboring Croatia (15), but differs from the results obtained in Taiwan where only $2.4 \%$ of the 211 investigated strains were susceptible to this antibiotic (31). High rates of susceptibility of $91 \%$ were observed for ampicillin and amoxicillin, which is in agreement with the reports by Matter and Kucerova $(19,22)$. On the other hand, these results differ from the results of Vanni (30) who found $69 \%$ and $82 \%$ resistance to ampicillin and amoxicillin, respectively. The susceptibility to gentamicin in our study was $77 \%$, which is much higher than susceptibil-

Tab. 1. Antimicrobial susceptibility of 148 isolates of $A$. pleuropneumoniae

\begin{tabular}{|l|c|c|c|c|c|c|}
\hline \multirow{2}{*}{ Antibiotics } & \multicolumn{2}{|c|}{ Susceptible } & \multicolumn{2}{c|}{ Intermediate } & \multicolumn{2}{c|}{ Resistant } \\
& No. isolates & $\%$ & No. isolates & $\%$ & No. isolates & $\%$ \\
\hline Ampicillin & 136 & 91.82 & 2 & 1.35 & 10 & 6.75 \\
Amoxicillin & 136 & 91.82 & 0 & 0 & 12 & 8.10 \\
Amoxicillin/clavulanic acid & 143 & 96.82 & 0 & 0 & 5 & 3.37 \\
Cefotaxime & 148 & 100.00 & 0 & 0 & 0 & 0 \\
Gentamicin & 114 & 77.02 & 17 & 11.48 & 17 & 11.48 \\
Streptomycin & 93 & 62.83 & 9 & 6.08 & 46 & 31.08 \\
Enrofloxacin & 148 & 100.00 & 0 & 0 & 0 & 0 \\
Lincospectin & 143 & 96.62 & 0 & 0 & 5 & 3.37 \\
Tetracycline & 89 & 63.13 & 8 & 5.40 & 51 & 34.45 \\
Florfenicol & 148 & 100.00 & 0 & 0 & 0 & 0 \\
Trimethoprim/sulfamethoxazole & 95 & 64.18 & 17 & 11.48 & 36 & 24.32 \\
\hline
\end{tabular}


ity rates of $5.7 \%$ and $43 \%$ published by other authors $(26,31)$. In our study the highest resistance rates were determined to tetracycline $(34 \%)$ and streptomycin $(31 \%)$. A significantly higher level of resistance to tetracycline was observed in Spain 85\% (14) as well as in Australia 75\% (9). Higher levels of resistance of $88.4 \%$ and $90.7 \%$ for chlortetracycline and oxitetracycline, respectively, were identified in Canada (17). When compared with our previous results (34), we observed a significant decrease in thetetracycline resistance from $53 \%$ to $34 \%$ as well as in trimethoprim/sulfamethoxazole resistance from $56 \%$ to $24 \%$. Similar results for sulfonamides were registered in the Czech Republic (25). A significant reduction of resistance for these two antimicrobial agents might have occurred as a result of their reduced usage during recent years, especially in treating animals from the category $15-40 \mathrm{~kg}$ of body weight. In several previous Serbian studies $(27,33,35)$ it was found that the most common respiratory bacterial pathogens in pigs were Pasteurella multocida, A. pleuropneumoniae and Haemphilus parasuis. Generally, in the treatment of infections caused by these pathogens tetracycline and trimethoprim/sulfamethoxazole are less efficient in comparison to other antibiotics, so this may partly explain the reduced usage of these two drugs in veterinary practice in Serbia. Of the total of 148 A. pleuropneumoniae tested isolates, $112(75 \%)$ showed resistance to one or more drugs. Seventy two isolates showed resistance to one drug, 24 to two, while 16 strains were resistant to three or more drugs. Among the 72 isolates, 34 were resistant to tetracycline, 19 to streptomycin, 14 to trimethoprim/sulfamethoxazole, 4 to ampicillin and one isolate was resistant to gentamicin. Patterns of co-resistance to the two drugs were as follows: 9 isolates were resistant to streptomycin and trimethoprim/sulfamethoxazole, 6 to gentamicin and tetracycline, 6 to gentamicin and streptomycin, one to ampicillin and amoxicillin, one to amoxicillin and trimethoprim/sulfamethoxazol, while one was resistant to amoxicillin/clavulanic acid and gentamicin. In this study, multidrug resistance (MDR) was considered as resistance to three or more antimicrobials. A total of 16 isolates showed multidrug resistance pattern (Tab. 2).

Tab. 2. Multidrug resistant (MDR) patterns detected in A. pleuropneumoniae clinica isolates

\begin{tabular}{|l|c|}
\hline \multicolumn{1}{|c|}{ MDR pattern } & Number of isolates \\
\hline Str, Tet, T/S & 6 \\
\hline Amp, Amx,Tet & 3 \\
\hline Gen, Str, Lsp & 3 \\
Amx, A/C, Str, Lsp, T/S & 1 \\
\hline Amx, A/C, Gen, Str, Lsp, T/S & 1 \\
\hline Amp, Amx, Gen, Str, Tet, T/S & 2 \\
\hline
\end{tabular}

Explanations: Str - streptomycin; Tet - tetracycline; T/S - trimethoprim/sulfamethoxazole; Amp - ampicillin; Amx - amoxicillin; Gen - gentamicin; Lsp - lincospectin; A/C - amoxicillin/ clavulanic acid
It is interesting, to note that all five strains resistant to lincospectin belonged to the multiresistant groups, as well as 6 of the 12 strains resistant to amoxicillin. Multidrug resistance of $A$. pleuropneumoniae strains has also been registered in other regions of the world. For example, in Canada five isolates demonstrated multidrug resistance to penicillins, streptomycin, tetracyclines and sulfanomides, whereas three others showed multiresistance to streptomycin, sulfonamides and tetracyclines (17). In many countries different levels resistance of $A$. pleuropneumoniae $(10,14,25$, $30)$ have been determined. Bacterial pathogens that cause diseases in pigs including A. pleuropneumoniae often demonstrate resistance to drugs commonly used in veterinary practice. Without knowledge of local resistance, use of antimicrobials creates a risk for treatment failure, but also for the spread of resistance among $A$. pleuropneumoniae isolates and the emergence of an unfavorable epizootic situation. Antimicrobial resistance is one of the major problems of human and veterinary medicine of the $21^{\text {st }}$ century. Continuous monitoring of antimicrobial resistance and responsible usage of drugs in livestock are very important in protecting against bacterial pathogens (23). Classes of antimicrobials such as cephalosporins and fluoroquinolones are relevant for human health and their use in livestock production should be limited or avoided as far as possible (1). Furthermore, antimicrobial usage in animals increases the possibility of acquiring resistance determinates in target bacteria as well as in commensal and zoonotic bacteria (2). There are numerous examples indicating that the level of bacterial resistance to antimicrobials depends of their consumption, and that consumption sooner or later leads to the development of resistance.

The results of the study showed high susceptibility rates of $A$. pleuropneumoniae clinical isolates to cefotaxime, enrofloxacin, florfenicol, amoxicillin/ clavulanic acid, lincospectin, ampicillin and amoxicillin. The highest resistance rates were established for tetracycline and streptomycin. It is important to emphasize that we noticed a decreasing trend in resistance to tetetracycline and trimethoprim/sulfamethoxazole. Continuous monitoring of antimicrobial susceptibility of $A$. pleuropneumoniae, as well as other respiratory bacterial pathogens, is important for prompt and adequate treatment of infected animals and prevention of the spread of resistance and reduces their current level.

\section{References}

1. Aarestrup F. M., Duran C. O., Burch G. S.: Antimicrobial resistance in swine production. Anim. Health Res. Rev. 2008, 9, 135-148.

2. Abatih E. N., Alban L., Ersboll A. K., Lofowong D. M.: Impact of antimicrobial usage on the transmission dynamics of antimicrobial resistant bacteria among pigs. J. Theoretic. Biol. 2009, 256, 561-573.

3.Archambault M., Harel J., Goure J., Tremblay Y. D. N., Jacques M.: Antimicrobial susceptibilities and resistance genes of Canadian isolates of Actinobacillus pleuropneumoniae. Microb. Drug Res. 2012, 18, 198-206.

4. Beddek A. J., Sheehan B. J., Bosse J. T., Kroll J. S., Langford P. J.: Two TonB Systems in Actinobacillus pleuropneumoniae: Their Roles in Iron Acquisition and Virulence. Infection Immunity 2004, 72, 701-708. 
5. Blackall P. J., Klaasen H. L., Bosh van den H., Kuhnert P., Frey J.: Proposal of a new serovar of Actinobacillus pleuropneumoniae: serovar 15. Vet. Microbiol. 2002, 84, 47-52.

6. Bosse J. T., Janson H., Sheehan B. J., Beddek A. J., Rycroft A. N., Kroll J. S., Langford $P$. R.: Actinobacillus pleuropneumoniae: pathobiology and pathogenesis of infection. Microbes Infection 2002, 4, 225-235.

7. Chung J. W., Jacques M., Coulton J. W.: Outer membrane proteins and iron uptake of Actinobacillus pleuropneumoniae, [in:] Khunert P., Christensen $\mathrm{H}$ (ed.): Pasteurellaceae, Biology, Genomics and Molecular Aspects. Caister Academic Press, Norfolk, UK 2008, p. 144-175.

8. Clinical and Laboratory Standards Institute (CLSI). Performance Standards for Antimicrobial Disk and Dilution Susceptibility Tests for Bacteria Isolated from Animals; Approved Standard Third edition. Clinical and Laboratory Standards Institute, Wayne, PA, USA, ISBN: 1-56238-660-3, CLSI Document M31-A3, 2008.

9. Dayao D. A., Gibson J. S., Blackall P. J., Turni C.: Antimicrobial resistance in bacteria associated with porcine respiratory disease in Australia. Vet. Microbiol. 2014, 171, 232-235

10. Dayao D. A. E., Dawson S., Kienzle M. J. P., Gibson J. S., Blackall P., Turni C. Variation in the Antimicrobial susceptibility of Actinobacillus pleuropneumoniae isolates in a pig, within a batch of pigs, and among batches of pigs from one farm. Microb. Drug Res. 2015, 21, 491-496.

11. Frey J., Bosse J. T., Chang Y. F., Cullen J. M., Fenwick B., Gerlach G. F. Gygi D., Haesebrouck F., Inzana T. J., Jansen R.: Actinobacillus pleuropneumoniae RTX-toxins: uniform designation of haemolysins, cytolysins, pleurotoxin and their genes. J. Gen. Microbiol. 1993, 139, 1723-1728.

12. Frey J., Kuhnert P., Viliger L., Nicolet J.: Cloning and characterization of an Actinobacillus pleuropneumoniae outer membrane protein belonging to the family of PAL lipoproteins. Res. Microbiol. 1996, 147, 351-361.

13. Gottschalk M., Taylor D. J.: Actinobacillus pleuropneumoniae, [in:] Straw B. E., Zimmerman J. J., D'allaire S, Taylor D. J.: Diseases of Swine. Blackwell Publishing, Oxford, UK 2006, p. 563-576.

14. Gutierrez-Martin C. B., Blanco N. G., Blanco M., Navas J., Rodriguez-Ferri $E$. F.: Changes in antimicrobial susceptibility of Actinobacillus pleuropneumoniae isolated from pigs in Spain during the last decade. Vet. Microbiol. $2006,115,218-222$.

15. Habrun B., Kompes G., Cvetnić Z., Spicic S., Benic M., MitakM.: Antimicrobial sensitivity of Escherichia coli, Salmonella spp, Streptococcus suis and Actinobacillis pleuropneumoniae isolated from diagnostic samples from large pig breeding farms in Croatia. Vet. Arhiv. 2010, 80, 571-583.

16. Haesebrouck F., Chiers K., Overbeke van, I., Ducatelle R.: Actinobacillus pleuropneumoniae infections in pigs: the role of virulence factors in pathogenesis and protection. Vet. Microbiol. 1997, 58, 239-249.

17. Jacobsen I., Henning-Pauka I., Baltes N., Trost M., Gerlach G. F.: Enzymes involved in anaerobic respiration appear to play a role in Actinobacillus pleuropneumoniae virulence. Infection Immunity 2005, 73, 226-234.

18. Jacques M.: Surface polysaccharides and iron-uptake systems of Actinobacillus pleuropneumoniae. Canad. J. Vet. Res. 2004, 68, 81-85.

19. Kucerova Z., Hradecka H., Nechvatalova K., Nedbalcova K.: Antimicrobia susceptibility of Actinobacillus pleuropneumoniae isolates from clinical out breaks of porcine respiratory diseases. Vet. Microbiol. 2011, 150, 203-206.

20.Lee K. E., Choi H. W., Kim H. H., Song J. Y., Yan D. K.: Prevalence and Characterization of Actinobacillus pleuropneumoniae isolated from Korean Pigs. J. Bacteriol. Virol. 2015, 45, 19-25.
21. Maldonado J., Valls L., Martinez E., Riera P.: Isolation rates, serovars, and toxin genotypes of nicotinamide adenine dinucleotide-independent Actinobacillus pleuropneumoniae among pigs suffering from pleuropneumonia in Spain. J.V.D.I. 2009, 21, 854-857.

22. Matter D. A., Rossano S., Limat L., Vorlet-Fawer I., Brodar D., Perreten V. Antimicrobial resistance profile of Actinobacillus pleuropneumoniae and Actinobacillus porcitonsillarum. Vet. Microbiol. 2007, 122, 146-156.

23. Merle R., Hajek P., Käsbohrer A., Hegger-gravenhorst C., Mollenhauer Y. Robanus M., Ungemach F., Kreienbrock L.: Monitoring of antibiotic consumption in livestock: A German feasibility study. Prev. Vet. Med. 2012, 104, 104

24. Morioka A., Asai T., Nitta H., Yamamoto K., Oqikubo Y., Takahashi T. Suzuki S.: Recent trends in antimicrobial susceptibility and the presence of the tetracycline resistance gene in Actinobacillus pleuropneumoniae isolates in Japan. J.V.M. S. 2008, 70, 1261-1264.

25. Nedbalcova K., Satran P., Jaglic Z., Ondriasova R.: Monitoring of antibiotic resistance in isolates of Actinobacillus pleuropneumoniae in the Czech Republic between 2001 and 2003. Vet. Med. Czech. 2005, 50, 181-185.

26. Pascu C., Costinar L., Herman V., Catana N., Serbescu M., Surpati A. Monitoring of antibiotic resistance in isolates of Actinobacillus pleuropneumoniae in the west part of Romania between 2005 and 2009. Lucr. Stiint. Med. Vet. 2010, 18, 173-176

27. Radanovic O.,Zutic J., Jakic-Dimic D., Kureljusic B., Savic B.: The prevalence of Pasteurella multocida from farm pigs in Serbia. Mac.Vet. Rev. 2016, 39, 139-142.

28. Shin M., Cha S., Lee W., Sung J., Roh Y., Yoo H.: Characterization of apxIVA of Actinobacillus pleuropneumoniae Korean isolate. Proc. $21^{\text {st }}$ IPVS Congress, Vancouver, Canada 2010, p. 604.

29. Tremblay Y., Deslandes V., Jacques M.: Actinobacillus pleuropneumoniae genes expression in biofilms cultured under static conditions and in a drip-flow apparatus. BMC Genomics 2013, 14, 364.

30. Vanni M., Merenda M., Barigazzi G., Garbarino C., Luppi A., Tognetti R. Intorre L.: Antimicrobial resistance of Actinobacillus pleuropneumoniae isolated from swine. Vet. Microbiol. 2012, 156, 172-177.

31. Yang C. Y., Lin C. N., Lin C. F., Chang T. C., Chiou M. T.: Serotypes, antimicrobial susceptibility, and minimal inhibitory concentrations of Actinobacillus pleuropneumoniae isolated from slaughter pigs in Taiwan (2002-2007). J. V. M. S. 2011, 73, 205-208.

32. Yoshimura H., Takagi M., Ishimura M., Endoh Y. S.: Comparative in vitro activity of 16 antimicrobial agents against Actinobacillus pleuropneumoniae. Vet. Res. Comm. 2002, 26, 11-19.

33. Zutic J., Vojinovic D., Radosavljevic V.: Seroprevalence of Actinobacillus pleuropneumoniae in swine originated from commercial farms in Serbia. Biotechnol. Anim. Husbandry 2014, 30, 295-302.

34. Zutic M., Asanin R., Milic N., Ivetic V., Vidic B., Zutic J., Asanin J.: Isolation and identification of Actinobacillus pleuropneumoniae in pigs lungs at farms and their sensitivity to antibiotics. Acta Vet. Belgrade 2008, 58, 499-507.

35. Zutic M., Ivetic V., Radanovic O., Zutic J., Jakic-Dimic D., Savic B., Pavlovic I. Stanojevic S.: Investigations of representation of certain bacteria strains in lungs of pigs with pneumonia. Vet. Glasnik 2009, 63, 3-15.

Corresponding author: Žutić Jadranka DVM, PhD, Scientific Institute of Veterinary Medicine of Serbia, ul. Vojvode Toze 14, 11000 Belgrade, Serbia; e-mail: jadranka.zutic@gmail.com 